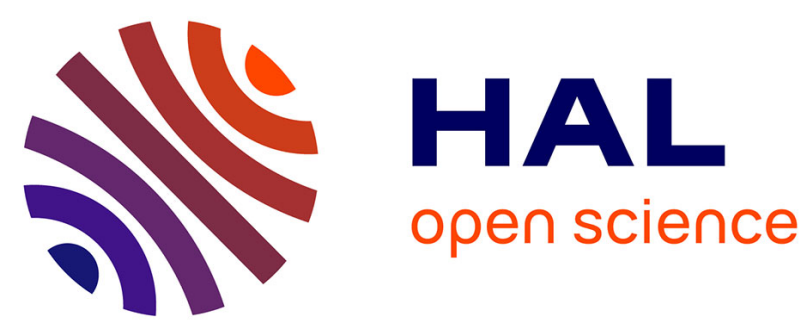

\title{
La médiation parentale, élément clé de la construction du sens de la pratique de l'argent de poche
}

\author{
Marcio Dias, Valérie Inés Demerson de La Ville
}

\section{To cite this version:}

Marcio Dias, Valérie Inés Demerson de La Ville. La médiation parentale, élément clé de la construction du sens de la pratique de l'argent de poche. Revue internationale de l'éducation familiale, 2015, Entre enfants et parents: la socialisation dans l'espace quotidien de la famille, 37, pp.63-85. 10.3917/rief.037.0063 . hal-01627594

\section{HAL Id: hal-01627594 https://hal.science/hal-01627594}

Submitted on 26 Oct 2018

HAL is a multi-disciplinary open access archive for the deposit and dissemination of scientific research documents, whether they are published or not. The documents may come from teaching and research institutions in France or abroad, or from public or private research centers.
L'archive ouverte pluridisciplinaire HAL, est destinée au dépôt et à la diffusion de documents scientifiques de niveau recherche, publiés ou non, émanant des établissements d'enseignement et de recherche français ou étrangers, des laboratoires publics ou privés. 


\title{
La médiation parentale, élément clé de la construction par l'enfant du sens de la pratique de l'argent de poche
}

\author{
Marcio Dias et Valérie-Inés de La Ville ${ }^{1}$
}

De nos jours, les enfants disposent de sommes relativement importantes d'argent de poche, argument souvent utilisé par les marques pour justifier le fait qu'elles s'adressent directement à eux. Afin de comprendre comment la pratique de l'argent de poche contribue à rendre les enfants autonomes dans leurs activités de consommation, nous avons mené une étude ethnographique pendant six mois au sein de 12 familles poitevines (France) d'élèves de classes de CM2 et de $6^{\text {ème }}$, en observant leurs interactions auprès de plus de 50 personnes. Cet article rend compte des dynamiques relationnelles sur lesquelles se construisent à la fois la signification de l'argent de poche au sein de la famille et ses implications normatives qui cadrent le niveau de participation de l'enfant à certaines activités de consommation.

Mots clés : argent de poche, famille, médiation parentale, autonomie de l'enfant, consommation enfantine.

Parental mediation, a key element in the construction of a child's understanding about the use of pocket money

Nowadays, children get a significant amount of pocket money, an argument often used by brands to justify the reason why they address them directly. In order to understand how the use of pocket money enables children to become autonomous in their consumption activities, we carried out a six-month ethnographic observation of twelve 10 to 11 year old children - pupils in the 5th year of primary school and the 1 st year of high school - within 12 French families living in Poitiers (France). Moreover, we observed their interactions with more than 50 people from different contexts in their everyday lives. This article analyzes the relational dynamics between parents and children which construct both the meaning of pocket money within the family and the normative the normative requirements that frame the degree of child participation in specific consumption activities.

Key words : pocket money, family, parental mediation, children's autonomy, child consumption.

La mediación parental, elemento clave de la construcción por el niño del sentido de la práctica del dinero de bolsillo

Hoy en día, los niños reciben importantes cantidades de dinero de bolsillo, un argumento a menudo utilizado por las marcas para legitimar que se dirigen directamente a ellos. Con el fin de entender cómo la práctica del dinero de bolsillo permite a los niños volverse autónomos en sus actividades de consumo, hemos llevado a cabo un estudio etnográfico durante seis meses con estudiantes de 10 a 11 años-alumnos del quinto año de escuela primaria y del primer año de instituto - de 12 familias francesas que viven en Poitiers (Francia). También hemos observado sus interacciones con 50 personas, que intervienen en diferentes aspectos de sus vidas. En este artículo, analizamos las dinámicas relacionales que involucran a padres e hijos a través de las cuales se construyen tanto el sentido del dinero de bolsillo en el seno de cada familia como los requisitos normativos que enmarcan el grado de participación de los niños en actividades de consumo específicas.

Palabras clave : dinero de bolsillo, familia, mediación parental,autonomía del niño, consumo infantil.

\footnotetext{
${ }^{1}$ Marcio Dias, docteur ès Sciences de Gestion de l'Université de Poitiers, Laboratoire CEREGE, EA 1722, Université de Poitiers, France.

Valérie-Inés de La Ville, Professeure de Sciences de Gestion, Directrice Centre Européen des Produits de l'Enfant, Laboratoire CEREGE EA 1722 et MSHS USR 3565, Université de Poitiers, France.

Contact : delaville@iae.univ-poitiers.fr
} 


\section{Introduction}

Selon l'étude Ipsos menée pour la banque ING auprès de 12000 familles européennes de 13 pays en $2014^{2}$, le montant moyen hebdomadaire accordé par les parents français est de $2 €$ avant 5 ans, $5 €$ entre 5 et 10 ans, $10 €$ entre 10 et 15 ans et $20 €$ au-delà. Un précédent sondage CSA de $2012^{3}$, révèle que $64 \%$ des jeunes de 14 à 17 ans reçoivent régulièrement de l'argent de poche et seulement 2 foyers sur 10 pensent en diminuer le montant en contexte de crise. Pour les enfants de 6 à 15 ans, une autre enquête datant de 2009 a établi que $40 \%$ des familles françaises accordaient une moyenne mensuelle de 18,90 euros à leurs enfants ${ }^{4}$. Il est alors possible d'évoquer l'existence d'un marché spécifique regroupant les dépenses liées à l'argent de poche (Filser, 1994) qui a un poids d'environ deux milliards d'euros annuels dans l'économie domestique française.

Les résultats d'un autre sondage ${ }^{5}$ montrent que $47 \%$ des parents interrogés affirment donner de l'argent de poche à leurs enfants «pour leur apprendre à gérer un budget ». Cette réponse signifie-t-elle que l'enfant n'a pas les compétences pour gérer seul un budget ou : au contraire, qu'il doit devenir réellement indépendant pour le faire tout seul ? Si s'engager dans une activité de consommation liée à l'argent de poche ne suffit pas pour maîtriser un budget, il faut alors se demander comment se met en place un processus d'apprentissage dans ce but.

Si $33 \%$ des parents accordant de l'argent de poche à leurs enfants déclarent que "cela permet aux enfants de s'acheter ce dont ils ont envie », l'imprécision du sens réel de cette pratique demeure... Cette autorisation estelle une liberté totale accordée aux activités de consommation et aux décisions

\footnotetext{
${ }^{2}$ ING International Survey, « Learning Young: Does getting pocket money teach saving habits for life ?, étude Ipsos publiée en septembre 2014 sur le site : http://www.ing.com/About-us/Our-stories/Features/Features/Pocket-money-boostsmoney-management-skills-in-adulthood.htm

${ }^{3}$ Sondage CSA «Les jeunes et l'argent de poche » sur l'argent de poche accordé aux enfants de 14 à 17 ans, réalisé du 6 au 27 Février 2012 et publié en septembre 2012 sur le site : www.csa.eu

${ }^{4}$ Nous faisons référence au sondage exclusif CSA pour le Crédit Agricole, Les parents et l'argent de poche des enfants, réalisé par téléphone du 6 au 11 juillet 2009 auprès de 501 parents d'enfants âgés de 6 à 15 ans publié sur le site : www.csa.eu

5 Sondage réalisé par le CSA pour le Crédit Agricole par téléphone du 4 au 7 juillet 2008 auprès de 503 parents d'enfants âgés de 6 à 15 ans publié sur le site : www.csa.eu.
} 
des enfants ? L'enfant ne fait-il que subir l'influence de valeurs familiales et sociales ? Comment les parents peuvent-ils être sûrs que leurs enfants ont acquis des repères socioculturels qui s'expriment dans leurs choix de consommation de leur propre volonté ?

Ces questionnements nous ont conduits à nous demander comment la pratique de l'argent de poche pouvait contribuer à l'autonomie de l'enfant dans ses activités de consommation. Conceptuellement, nous considérons que l'autonomisation constitue une construction progressive dont le décryptage ne doit pas être centré sur l'enfant, mais sur les interactions qu'il établit avec son entourage. C'est pourquoi nous avons centré notre problématique sur les formes d'interaction familiale qui contribuent à la pratique d'argent de poche. Nous analysons comment cette pratique transforme les normes de participation de l'enfant aux activités de consommation afin de cadrer son niveau de participation en fonction du contexte (De La Ville et Tartas, 2008).

Dans la première partie de cet article, nous précisons les concepts relatifs à la pratique de l'argent de poche pour éclairer son rôle de facteur d'automisation de l'enfant. Ensuite, nous proposons une méthode qui prend en considération la dynamique du rapport intersubjectif parents/enfants dans les interactions établies pour utiliser l'argent de poche dans des activités de consommation. Dans la troisième partie, nous analysons le rôle des parents et la façon dont la pratique de l'argent de poche met sous tension leur projet éducatif. On pourra en conclure que l'attribution d'argent de poche à l'enfant constitue une stratégie d'éducation destinée à prévenir le risque d'une consommation débridée...

\section{L'argent de poche comme facteur d'autonomisation de l'enfant consommateur : précisions conceptuelles}

Même si pour la plupart des auteurs, l'argent de poche est un indicateur du statut de l'enfant au sein de la famille (Octobre, 2004), le concept d'autonomie de l'enfant en matière de consommation reste encore flou. Cette autonomie s'acquiert-elle par la simple possession d'argent et de responsabilités ou fait-elle aussi référence à la gestion de cet argent ? Les parents ne joueraient-ils d'autre rôle que celui de pourvoyeurs d'argent de poche?

Le statut de consommateur de l'enfant peut-il être uniquement centré sur la notion de "maturité économique », comme nous le propose Roland-Lévy (2005, p. 61) ? De nombreux auteurs dans les champs du Marketing et de la 
Psychologie Économique considèrent l'attribution d'argent de poche comme une émancipation (McNeal, 1992, 2007 ; Filser, 1994 ; Furnham, 1999 ; Barnet-Verzat et Wolff, 2001 ; Heilbrunn, 2004 ; Roland-Lévy, 2005 ; Vulbeau, 2007). Il apparaît comme un signe d'indépendance économique qui permet aux enfants de vivre des expériences favorisant l'acquisition de règles relatives à la consommation. C'est pourquoi, consultants et chercheurs en marketing affirment que, pour éduquer les enfants à la consommation, il faut : leur donner un montant d'argent qui ne soit pas négligeable et qu'ils puissent dépenser à leur guise ; qu'il soit donné régulièrement à l'enfant; une partie de cet argent doit faire l'objet d'une démarche d'épargne, pour que les enfants comprennent que tous leurs désirs ne peuvent pas être satisfaits de façon instantanée (Siegel, Coffey et Livingston, 2002 ; Brée, 1993 ; Marshall et Magruder, 1960).

Deux conceptions de l'autonomie sous-tendent cette façon d'aborder l'enfant consommateur. Premièrement, une idéologie qui assigne aux individus le droit à l'autodétermination : en tant qu'acteur social, l'enfant possède le droit de s'exprimer et est incité à manifester un désir devant être respecté par les adultes. Ainsi, Marshall et Magruder (1960), préconisent de donner à l'enfant une somme d'argent considérable qu'il puisse dépenser à sa guise. Deuxièmement, l'autonomie est entendue au sens normatif, comme une capacité empirique de sujets concrets à déterminer leur vie sans contrainte. Dans ce cas, les rapports sociaux ne doivent pas s'opposer aux capacités des enfants de dépenser leur argent de poche " pour tout ce qu'ils désirent, quand ils le souhaitent, où ils veulent » (McNeal 1992, p. 34). Il nous semble que ces cadres théoriques opèrent une réduction majeure de l'autonomie de l'enfant en la limitant à l'indépendance et à l'individualisation de l'enfant. L'application de tels concepts aux pratiques de consommation de l'enfant n'est cependant pas exempte de quelques contradictions. En effet, la manifestation d'un désir doit être analysée à partir des rapports sociaux qui le suscitent et grâce auxquels celui-ci peut se réaliser. D'un point de vue normatif, cet enfant capable de déterminer sa vie «sans contrainte », n'est que la représentation théorique d'un sujet épistémique. La figure de l'enfant consommateur que cette approche construit privilégie la mise en scène d'une individualité cognitive en-dehors de relations sociales, plutôt qu'une individuation progressive dans le cadre de l'évolution d'une multiplicité de rapports sociaux réels.

De façon plus nuancée que ces travaux, Gentina (2008) montre que l'autonomie des adolescents se constitue progressivement en s'appuyant sur l'ensemble des relations du réseau de ses groupes sociaux. Elle en conclut que 
l'autonomie et le besoin d'intégration doivent être considérés comme deux éléments complémentaires agissant au cœur de la dynamique de la transformation adolescente.

Selon le philosophe Axel Honneth (2008), c'est à partir de la quête de reconnaissance de l'individu, structurée autour des attentes normatives suscitées et façonnées par les interactions sociales auxquelles il participe, que l'autonomie se manifeste. On peut alors parler d'autonomie décentrée (Honneth, 2008) ou relationnelle (Mackenzie et Stoljar, 2000). Par contraste avec les principes d'autonomie personnelle issus de l'approche kantienne, cette conception décentrée souligne la construction partagée de l'autonomie à partir de règles co-élaborées dans les rapports sociaux. Cette approche prend en compte la dynamique intersubjective qui permet aux sujets en interaction de construire une signification partagée de l'activité dans laquelle ils sont engagés afin que cette dernière puisse se poursuivre.

\section{Une méthode d'inspiration ethnographique pour observer la pratique de l'argent de poche au sein des familles}

Retenir cette perspective relationnelle du processus d'autonomisation de l'enfant consommateur implique d'élaborer une méthode de recherche permettant d'analyser les activités de consommation pour lesquelles l'enfant utilise son argent de poche. Il s'agit d'étudier comment l'enfant développe son autonomie en matière de consommation au sein même d'un ensemble de relations médiatisées à la fois par différents acteurs et par un ensemble d'outils culturels mis à sa disposition (De La Ville et Tartas, 2011).

Selon Martens, Southerton et Scott (2004), les parents exercent l'influence la plus importante sur l'enfant dans ses acquisitions relatives à la consommation : ils jouent un rôle de filtre - gatekeeper - sur les produits qui peuvent être consommés dans leur foyer et participent à instaurer une culture de consommation familiale qui agit dans le processus de développement des jeunes consommateurs. En étudiant le rapport entre la communication familiale et le comportement de consommation de l'enfant, Gollety et Gautellier (2001) dressent une typologie du dialogue entre enfants et parents qui différencie cinq modalités d'apprentissage : par discussions informelles à la maison; par la médiation d'informations extérieures ; par la préparation et l'analyse des achats

autonomes de l'enfant ; par des discussions sur le lieu des achats ; par une combinaison singulière de ces différentes modalités d'apprentissage. 
Ces travaux considèrent que les parents développent un type de communication relativement uniforme avec leurs enfants. Or, comme le suggèrent Montandon et Saloni (2002), les différences de développement des enfants d'une même fratrie s'expliqueraient par des variations dans le style de communication affective et éducative des parents soucieux de s'adapter au comportement singulier de chaque enfant : l'enfant n'est donc pas passif car il interprète les expériences qu'il vit et se montre capable de développer des stratégies pour transformer ces rapports familiaux. Enfin, l'effet des styles éducatifs des parents peut être réduit, amplifié ou annulé par les interactions de l'enfant avec ses proches.

En poursuivant dans cette voie, nous nous sommes intéressés aux dynamiques communicationnelles par lesquelles l'enfant et ses parents négocient les conditions de la pratique d'argent de poche. Nous avons approché la vie quotidienne et les activités de consommation de 12 familles d'élèves de CM2 et $6^{\text {ème }}$ habitant Poitiers (France) grâce à une méthode ethnographique d'observation-participante de longue durée - de 3 à 4 heures tous les 15 jours pendant six mois (de janvier à juin 2010). (voir tableau1) Nous sommes également allés faire les courses avec les familles. Afin d'inclure dans notre analyse les interactions au sein du groupe familial ainsi que les activités ordinaires de consommation du groupe d'enfants étudié, nous avons veillé à inclure à travers différents types d'entretiens, plus de 50 personnes (parents, enseignants, fratrie, pairs, grands-parents, vendeurs, etc.) dans notre étude.

Pour réduire l'abondant matériau empirique recueilli, nous avons adapté les principes de la perspective historico-culturelle (Vygotski, 1978) et retenu trois axes d'interprétation :

1) en suivant la proposition faite par Léontiev (1978), nous avons distingué trois dimensions : l'activité, les actions et les opérations. La première dimension est liée à la motivation à s'engager dans l'activité ; la deuxième inclut les actions qui permettent à l'individu d'activer la motivation nécessaire pour s'engager dans des activités; et la troisième analyse les structures d'opérations grâce auxquelles diverses actions sont accomplies.

2) nous avons classé les niveaux de participation de l'enfant dans l'activité de consommation en distinguant les rôles de «participant périphérique légitime » par opposition à la figure de «participant central »

Tableau 1 - Le profil des familles enquêtées (Source : Márcio Dias, 2012)

\begin{tabular}{|l|c|c|c|c|c|}
\hline Famille & $\begin{array}{c}\text { Composition } \\
\text { de la famille }\end{array}$ & $\begin{array}{c}\text { Profession } \\
\text { des parents }\end{array}$ & $\begin{array}{c}\text { Enfant } \\
\text { classe }\end{array}$ & Sexe & $\begin{array}{c}\text { Argent } \\
\text { (euros) }\end{array}$ \\
\hline
\end{tabular}




\begin{tabular}{|c|c|c|c|c|c|}
\hline Les $\mathrm{S}$ & $\begin{array}{l}\text { Traditionnelle } \\
2 \text { frères }\end{array}$ & $\begin{array}{l}\text { Mère : Femme au foyer } \\
\text { Père : Comptable }\end{array}$ & $\begin{array}{l}\mathrm{XS} \\
\mathrm{CM} 2\end{array}$ & M & 16 \\
\hline Les $\mathrm{G}$ & $\begin{array}{l}\text { Recomposée } \\
1 \text { frère }\end{array}$ & $\begin{array}{l}\text { Mère : Professeure } \\
\text { Père : Technicien } \\
\text { informatique }\end{array}$ & $\begin{array}{l}\mathrm{TG} \\
\mathrm{CM} 2\end{array}$ & M & 5 \\
\hline Les $\mathrm{N}$ & $\begin{array}{l}\text { Recomposée } \\
1 \text { frère }\end{array}$ & $\begin{array}{l}\text { Mère : Avocate } \\
\text { Père : Avocat }\end{array}$ & $\begin{array}{l}\mathrm{KN} \\
\mathrm{CM} 2\end{array}$ & $\mathrm{~F}$ & 8 \\
\hline Les Q & $\begin{array}{l}\text { Traditionnelle } \\
\text { Enfant unique }\end{array}$ & $\begin{array}{l}\text { Mère : Administratrice } \\
\text { d'entreprise } \\
\text { Père : Professeur }\end{array}$ & $\begin{array}{l}\mathrm{BQ} \\
\mathrm{CM} 2\end{array}$ & $\mathrm{~F}$ & 5 \\
\hline Les $\mathrm{H}$ & $\begin{array}{l}\text { Recomposée } \\
2 \text { sœurs }\end{array}$ & $\begin{array}{l}\text { Mère : Administratrice } \\
\text { Père : Administrateur } \\
\text { d'entreprise }\end{array}$ & $\begin{array}{l}\mathrm{NH} \\
\mathrm{CM} 2\end{array}$ & $\mathrm{~F}$ & 5 \\
\hline Les $\mathrm{T}$ & $\begin{array}{l}\text { Traditionnelle } \\
2 \text { sœurs }\end{array}$ & $\begin{array}{l}\text { Mère : Infirmière } \\
\text { Père : Infirmier }\end{array}$ & $\begin{array}{l}\mathrm{BT} \\
\mathrm{CM} 2\end{array}$ & $\mathrm{~F}$ & 8 \\
\hline Les Z & $\begin{array}{l}\text { Traditionnelle } \\
1 \text { frère }\end{array}$ & $\begin{array}{l}\text { Mère : Opticienne } \\
\text { Père : Administrateur }\end{array}$ & $\begin{array}{l}\mathrm{JZ} \\
6^{\text {ème }}\end{array}$ & M & 10 \\
\hline Les $\mathrm{M}$ & $\begin{array}{l}\text { Traditionnelle } \\
1 \text { sœur / } 2 \text { frères }\end{array}$ & $\begin{array}{l}\text { Mère : Professeure } \\
\text { Père : Professeur }\end{array}$ & $\begin{array}{l}\mathrm{NM} \\
6^{\mathrm{ème}}\end{array}$ & M & 12 \\
\hline Les D & $\begin{array}{l}\text { Monoparentale } \\
2 \text { frères }\end{array}$ & Mère : Professeure & $\begin{array}{l}\text { QD } \\
6^{\text {ème }}\end{array}$ & M & 10 \\
\hline Les $\mathrm{N}$ & $\begin{array}{l}\text { Recomposée } \\
1 \text { soeur / } 1 \text { frère }\end{array}$ & $\begin{array}{l}\text { Mère : Administratriceur } \\
\text { Père : Pharmacien }\end{array}$ & $\begin{array}{l}\mathrm{DN} \\
6^{\text {ème }}\end{array}$ & $\mathrm{F}$ & 5 \\
\hline Les $\mathrm{C}$ & $\begin{array}{l}\text { Monoparentale } \\
1 \text { frère }\end{array}$ & Mère : Comptable & $\begin{array}{l}\mathrm{FC} \\
6^{\text {ème }}\end{array}$ & $\mathrm{F}$ & 12 \\
\hline Les $\mathrm{R}$ & $\begin{array}{l}\text { Traditionnelle } \\
3 \text { sœurs }\end{array}$ & $\begin{array}{l}\text { Mère : Professeure } \\
\text { Père : Bibliothécaire }\end{array}$ & $\begin{array}{l}\text { NR } \\
6^{\text {ème }}\end{array}$ & $\mathrm{F}$ & 8 \\
\hline
\end{tabular}

(Rogoff, Moore, Najafi, Dexter, Correa-Chávez et Solis, 2007 ; Rogoff, Matuso et White, 1996) ; et 
3) afin de repérer comment les enfants intériorisent le sens des activités de consommation liées à l'argent de poche, nous avons mis en lumière les médiateurs et médiations qui leur permettaient d'atteindre les résultats observés (repérer les produits ; choisir un produit ; s'informer sur le lieu de vente ; payer les achats effectués etc.)

En prenant les dimensions de l'activité comme catégories d'analyse, les données récoltées ont été réduites par un système de fiche de synthèse. Nous avons élaboré plus de cinquante tableaux décomposant de façon détaillée les trois niveaux des activités de consommation observées au sein de la famille. En outre, nous avons qualifié pour chacune de ces activités de consommation observées, quel était le niveau de participation de l'enfant à l'interaction et quels avaient été les médiateurs mobilisés pour cadrer ce niveau de participation.

Cette analyse minutieuse (voir Figure 1) nous a permis de mettre en valeur les divers outils de médiation mobilisés au cours des différentes activités de consommation et de nuancer les niveaux de participation des enfants à l'utilisation de l'argent de poche.

Figure 1 : Exemple de tableau d'analyse d'une activité de consommation (Source : Dias, Márcio, 2012)

\begin{tabular}{|c|c|c|c|c|}
\hline \multicolumn{3}{|c|}{ Décomposition de l'activité } & \multicolumn{2}{|c|}{ Décomposition de l'interaction } \\
\hline Activité & Action & Opération & Participation & Médiation \\
\hline \begin{tabular}{|l|} 
Avoir de \\
nouveaux \\
habits \\
Gagner de \\
l'argent \\
Vivre le rôle de \\
consommateur
\end{tabular} & $\begin{array}{l}\text { Négocier } \\
\text { avec les } \\
\text { parents } \\
\text { Payer } \\
\text { l'article } \\
\text { Sélectionner } \\
\text { des articles }\end{array}$ & $\begin{array}{l}\begin{array}{l}\text { Suivre les } \\
\text { suggestion } \\
\text { des parents }\end{array} \\
\text { Garder la } \\
\text { monnaie } \\
\text { S'informer } \\
\text { dans le } \\
\text { magasin }\end{array}$ & $\begin{array}{l}\text { Périphérique } \\
\text { payer le produit } \\
\text { avec l'aide des } \\
\text { parents } \\
\text { Centrale } \\
\text { payer le produit } \\
\text { sans aide ou } \\
\text { imitation }\end{array}$ & $\begin{array}{l}\text { Parents } \\
\text { Argent } \\
\text { Pairs } \\
\text { Médias } \\
\text { Produits } \\
\text { Magasins } \\
\text { Vendeurs } \\
\text { Ecole } \\
\text { Etc. }\end{array}$ \\
\hline
\end{tabular}

Comme technique d'élaboration et de vérification de nos résultats, nous avons retenu le principe général de la Théorie Ancrée (Glaser et Strauss, 2010), c'est-à-dire que nous avons établi des comparaisons systématiques pour reconstruire le sens que les protagonistes donnent à l'utilisation de l'argent de poche. Nous nous sommes centrés sur les significations que l'enfant élabore à 
partir des formes d'interaction et de guidage lui ayant permis de s'engager dans les actions et les opérations nécessaires à la réussite de ses pratiques situées de consommation. Ensuite, pour chaque activité analysée, nous sommes retournés sur le terrain pour vérifier avec les enfants si nous avions bien compris leur façon de construire le sens de ces activités de consommation. Sur les six mois d'observation, nous avons repéré les changements de raisonnement et de comportement de l'enfant à différents moments et dans divers espaces marchands conduisant à de nouvelles justifications de la pratique de l'argent de poche.

Cette comparaison des tableaux d'analyse et le retour régulier sur le terrain avec les enfants dans l'univers marchand (magasins et sites internet) nous ont permis de constater que les activités de consommation effectuées par les enfants ne doivent pas être comprises comme l'expression de capacités sociocognitives définitivement acquises par les enfants qui leur permettraient de pratiquer une consommation « indépendante » en fonction de critères qu'ils auraient librement élaborés.

En validant nos interprétations avec chaque enfant par différents retours sur le terrain en sa compagnie ${ }^{6}$, nous avons pu constater que les enfants sont réellement acteurs de leur propre processus d'apprentissage, ce qui les conduit à reconstruire en permanence le sens des activités de consommation liées à l'argent de poche : ils deviennent capables de certains gestes autonomes, mais ils sont bien loin de se considérer comme des consommateurs pleinement libres. Ils ont souligné à quel point les outils culturels mis à leur disposition les ont fortement guidés dans l'utilisation de leur argent de poche.

\section{Quel rôle les parents jouent-ils dans la pratique de l'argent de poche?}

À travers des interviews approfondies avec les parents sur la façon dont ils justifiaient la pratique de l'argent de poche, nous avons analysé la dynamique du rapport intersubjectif établi entre parents et enfants qui rend possible la participation de l'enfant à des activités de consommation en lien avec l'argent de poche. Afin de déceler les raisons ayant conduit les parents et les enfants à s'engager dans ces pratiques de consommation, nous avons approfondi les rapports entre parents et enfants justifiant de mobiliser l'argent de poche dans la réalisation des activités, actions et opérations de consommation. Dans ce but,

6 Lors de cette phase de notre travail, nous avons appliqué les principes méthodologiques du least-adult énoncés par Nancy Mandell (1988). 
nous avons appliqué les principes de l'intersubjectivité développés par Wersch (1984) qui rappelle qu'au cours de la réalisation d'une tâche, les parents peuvent : faire répéter à l'enfant les actions et opérations nécessaires pour réaliser la tâche; ou bien laisser l'enfant faire à sa manière et intervenir ensuite; soit encore, réaliser ensemble la tâche, en interagissant à partir des différents points de vue exprimés au cours de l'activité.

Toutefois, bien que les inter-actants d'une activité se situent dans un même contexte spatiotemporel, Wertsch (1984) souligne que cela ne signifie pas pour autant qu'ils développent une perception semblable de l'activité, puisque leur manière de ressentir le contexte dépend de la signification donnée par chacun à cet échange symbolique. Pour comprendre si la relation qui lie les interactants les conduit à partager une même représentation du contexte, c'est-àdire crée un rapport d'intersubjectivité, l'auteur propose d'analyser le mode d'action des inter-actants.

L'analyse de ces modes d'action nous a permis de dresser une première typologie à caractère exploratoire des rapports intersubjectifs entre parents et enfants à propos de l'utilisation de l'argent de poche.

Les rapports pro-actifs (quatre groupes familiaux)

Dans cette catégorie nous avons regroupé des groupes familiaux engagés de façon volontariste dans la pratique de l'argent de poche. Les parents estiment que la pratique de l'argent de poche peut permettre à l'enfant d'acquérir davantage d'expérience afin d'être en mesure de participer de manière mature et autonome à la société de consommation. Ils se fixent des objectifs relativement ambitieux en matière d'éducation à la consommation car ils considèrent l'argent de poche comme un outil pédagogique. Il nous semble important de souligner que, pour ces parents, aider leur enfant à « utiliser son argent de poche » est un projet éducatif complexe qui les conduit à prendre l'initiative d'attribuer de l'argent de poche à leur enfant et à l'accompagner tout au long de cet apprentissage de gestion de son budget.

Nous avons observé ce modèle d'action dans les comportements de la mère de $\mathrm{FC}$ (élève de $6^{\text {ème }}$, âgée de 11 ans), qui n'a pas hésité à proposer à sa fille de payer sa participation pour l'achat d'un cadeau collectif destiné à un professeur. En prenant le temps de lui expliquer la fonction pratique de l'argent de poche, la mère de la collégienne en a profité pour assigner un sens moral et affectif aux pratiques de consommation. Dans la famille de QD (élève de $6^{\text {ème}}$, âgé de 12 ans), c'est sa mère qui l'engage à se renseigner sur le prix d'une 
console de jeu, pour réunir les éléments d'information nécessaires pour prendre conjointement la décision d'achat.

Ainsi, dans le cadre de rapports pro-actifs, c'est le sens proposé par les parents quant à l'utilisation de l'argent de poche, qui va servir de base pour l'élaboration conjointe de significations suffisamment partagées pour rendre possible la participation de l'enfant à des activités de consommation.

Les rapports réactifs (deux groupes familiaux)

Au sein des groupes familiaux que nous avons regroupés dans cette catégorie, les parents attendent en général que l'initiative en matière d'argent de poche vienne de leurs enfants. Ils considèrent que le fait d'apprendre à gérer son propre argent peut aider l'enfant à grandir, mais ce n'est pas pour autant qu'ils vont créer des situations qui stimulent la participation effective de l'enfant à des activités de consommation. L'utilisation de l'argent de poche n'entre pas dans les pratiques ordinaires ou quotidiennes de l'enfant et, de ce fait, cette pratique reste relativement marginale ou exceptionnelle dans la vie de l'enfant car ses parents assument toujours la responsabilité de payer les produits achetés. Se met alors en place une situation particulière puisque l'argent de poche que possède l'enfant peut ne jamais être sollicité pour des achats concrets.

Le rapport entre $\mathrm{KN}$ (élève de CM2, âgée de 10 ans) et ses parents à propos de l'argent de poche reflète ce modèle d'action réactif. Pendant les six mois d'observation, $\mathrm{KN}$ n'a pas dépensé son argent vu qu'elle ne l'emportait jamais avec elle. Ce qui ne signifie pas qu'elle s'est trouvée exclue du monde marchand : elle a fait les soldes, elle a acheté des jeux et des souvenirs et ses parents ont toujours accédé à ses désirs en payant ses achats. Mais KN n'a pas été incitée à dépenser son propre argent de poche et n'a pas non plus été guidée par ses parents pour apprendre à bien l'utiliser.

Les rapports réactifs ne conduisent pas à mettre en valeur l'utilisation que l'enfant est susceptible de faire de l'argent de poche qui lui est accordé, ce qui crée une situation où le partage de significations entre parents et enfants à propos de la pratique de l'argent de poche est extrêmement réduit, ce qui ne permet pas à l'enfant de développer son autonomie en matière de consommation. Le manque d'initiative des enfants de ce groupe conduit à très peu de situations réelles d'usage de leur argent de poche qui a pour eux une autre signification - un trésor, voire un jouet qui reste dans leur chambre. 
Les rapports défensifs (trois groupes familiaux)

Les parents qui établissent ce type de rapport avec leurs enfants ont trouvé, dans la pratique de l'argent de poche, une solution pour éluder les demandes constantes de l'enfant en matière d'achats. Les parents réunis dans ce groupe voient l'argent de poche comme une opportunité pour amener l'enfant à mieux comprendre la valeur de l'argent et à auto-contrôler ses envies d'achats en l'encourageant à se poser un certain nombre de questions préalables. Par la confrontation des pulsions d'achat de leur enfant au montant limité d'argent de poche qui lui est accordé, les parents esquivent la pression constante de leur enfant en matière de consommation : "Maintenant que tu as ton propre argent, tu t'achètes ce que tu veux avec cet argent $»$. Cette attitude place l'enfant dans une situation de contrainte et l'oblige à hiérarchiser ses envies ou bien à renoncer à certains de ses désirs.

Chez XS (élève de $\mathrm{CM} 2$, âgé de 11 ans), les discussions relatives à l'utilisation de l'argent de poche servent de mécanisme de défense pour contrôler les demandes constantes de l'enfant. Ce conditionnement est manifeste chez ce garçon, quand il se plaint : «Maintenant que j'ai de l'argent de poche, mes parents me disent toujours «non! "quand je souhaite qu'ils payent mes achats ».

Dans le cas de rapports défensifs, c'est souvent le sens peu réaliste (selon les parents) que l'enfant donne à l'argent qui conduit les parents à tenter de construire une signification partagée de l'argent de poche soulignant la contrainte liée à son montant limité plutôt que les possibilités d'exploration de l'univers marchand qu'il permet.

Le rapport idéologique ou critique (trois groupes familiaux)

Dans ces rapports, l'argent a pour objectif d'apprendre à l'enfant à réfléchir avant de dépenser, tout en faisant la différence entre « ce qu'il veut » et «ce que les autres veulent qu'il ait». Les parents considèrent que le fait d'accorder de l'argent de poche à l'enfant doit conduire ce dernier à construire son identité en partie en-dehors de la société de consommation, sur un système de valeurs qui lui soit propre et qui ne lui soit pas imposé par les structures marchandes. Cette démarche idéologique amène les parents à entretenir un dialogue continu sur des questions éthiques, morales et identitaires liées à la consommation. Ils soulignent la contradiction entre le désir d'être en conformité avec les normes du groupe de pairs et le besoin de se construire une identité propre hors de certaines normes sociales. Lorsqu'une situation de 
consommation est initiée au sein du groupe de pairs de l'enfant, ils s'impliquent dans le dialogue et invitent leur enfant à réfléchir : «Est-ce que tu as vraiment besoin de ça? Pourquoi tu veux acheter ça? Est-ce que tu sais que ce produit a été fabriqué en Chine, à partir d'exploitation du travail des enfants ?»...

Les parents de TG (élève de CM2, âgé de 10 ans) n'ont pas laissé passer l'opportunité d'expliquer à leurs fils que les jouets en plastique qu il voulait s'acheter reposaient sur l'exploitation du travail d'enfants chinois et qu'ils contribuaient à augmenter le taux de pollution par les plastiques. "J'ai eu des cauchemars avec ces petits enfants exploités », révèle TG quelques jours après avoir discuté avec ses parents. NM (élève de $6^{\text {ème }}$, âgé de 12 ans), même après avoir réuni toutes les conditions nécessaires pour s'acheter la paire de chaussures à la mode qu'il désirait, n'a finalement pas concrétisé son achat. «Je n'ai pas besoin de grand-chose », confirme NM.

Dans le cadre de rapports critiques, les parents et les enfants établissent, principalement à partir d'un regard interrogateur sur la consommation, une signification commune à propos de l'utilisation de l'argent de poche.

Figure 2 : Typologie des rapports intersubjectifs entre parents et enfants à propos de l'utilisation de l'argent de poche. Source : Dias, Márcio (2012)

\begin{tabular}{|cc|c|c|}
\hline \multicolumn{3}{c|}{ Mode d'action des pères/mères } \\
& \multicolumn{2}{c|}{ Initie l'action } & \multicolumn{2}{c|}{ Agit après } \\
\cline { 3 - 4 } $\begin{array}{c}\text { Mode } \\
\text { d'action } \\
\text { de } \\
\text { l'enfant }\end{array}$ & Agit & Type & Type \\
après & pro-actif & réactif & \\
\cline { 3 - 4 } & Initie & Type & Type \\
l'action & idéologique & défensif & \\
\hline
\end{tabular}

Cette typologie (figure 2) qui, dans une perspective vygotskienne, distingue différents rapports intersubjectifs entre parents et enfants permettant de construire une signification suffisamment partagée pour mettre en place une pratique de consommation liée à l'argent de poche, est de portée limitée puisqu'elle est fondée sur la relation approfondie que nous avons construite 
avec 12 familles poitevines.

\section{L'utilisation de l'argent de poche, une mise en tension du projet éducatif}

Ce résultat de notre recherche doit être mis en regard de l'abondante littérature sur les styles d'éducation parentaux. En effet, l'approche typologique des styles parentaux de Baumrind (1971) introduite dans les années 1970 a été utilisée à plusieurs reprises pour démontrer le lien entre le type de relations familiales et divers aspects du développement de l'enfant. Baumrind a caractérisé quatre styles parentaux en construisant une typologie des styles parentaux autour de deux dimensions, les demandes et les réponses parentales :

- «autocratique », avec des niveaux élevés de contrôle et de faibles niveaux de sensibilité ; les parents fixent des limites tout en répondant aux besoins des enfants :

- «permissif », combinant de faibles niveaux de contrôle et de hauts niveaux de sensibilité, sans avoir recours à la discipline ;

- «autoritaire », avec des niveaux élevés de contrôle parental du respect des règles familiales ;

- «négligent », caractérisé par un manque de contrôle et de sensibilité. Ce sont des parents qui, pour des raisons diverses, ont délaissé leurs fonctions parentales.

Cette modélisation a mis en exergue le rôle actif des enfants dans leur propre processus de développement à travers leur capacité à interagir et à influencer leurs parents (Darling et Steinberg, 1993). En ce qui concerne plus particulièrement l'influence des styles parentaux sur les pratiques de consommation des enfants, les travaux de Moschis (1985) ont abouti à la typologie suivante :

- la famille «laissez-faire » qui démontre peu d'intérêt pour l'harmonie familiale et dont les pratiques de communication sont presque inexistantes entre les parents et les enfants ;

- la famille «pluraliste » qui se caractérise par une communication ouverte des idées et au sein de laquelle l'enfant est encouragé à explorer de nouvelles idées et à s'exprimer ; 
- la famille «consensuelle » donne de la valeur à la découverte de nouvelles idées, cependant, cela ne peut pas s'opposer à la hiérarchie et à l'harmonie familiale ;

- la famille «protectrice » pour qui les valeurs centrées sur l'obéissance et l'harmonie familiale sont primordiales, laissant peu de place à l'enfant pour exprimer ses opinions personnelles.

Notre typologie rejoint en partie celle-ci. Les familles dites «pluralistes» peuvent entretenir des rapports pro-actifs avec leurs enfants à propos de l'argent de poche. Mais les rapports défensifs, par exemple, ne sont pas forcément le fait d'une famille «laissez-faire » car nous avons trouvé une préoccupation éducative forte de la part des parents.

Notre typologie souligne plutôt l'importance de la formation d'un espace intersubjectif qui permette conjointement aux parents et aux enfants d'explorer les signifiés associés à l'utilisation de l'argent de poche. Ces nouvelles significations diffèrent à la fois de la représentation donnée initialement par les parents, mais aussi de celles que les enfants élaborent activement. En effet, au cours du processus d'utilisation de l'argent de poche, les parents constituent l'agent de médiation principal pour que l'enfant s'essaye à des activités de consommation. Ce processus de médiation sémiotique, qui inclut le dialogue, la réflexion, les propositions des parents, l'image qu'ils convoquent du consommateur compétent etc., joue un rôle fondamental dans la socialisation de l'enfant aux univers de consommation.

Toutefois, ce rôle médiateur des parents dans l'apprentissage de la consommation par les enfants de CM2 et de $6^{\text {ème }}$ demande à être nuancé selon la nature des rapports intersubjectifs entre parents et enfants.

Dans les rapports "pro-actifs», les parents se montrent ouverts aux expériences de consommation faites par leurs enfants, tout en guidant ces derniers dans de nouvelles pratiques de consommation liées à l'argent de poche. Tout comme dans les rapports de type «idéologique ». Dans ces contextes, l'enfant valorise le fait d'être guidé par ses parents et de ce fait, l'argent de poche constitue un outil privilégié d'échange entre les parents et les enfants pour apprendre à confronter leurs désirs à leurs besoins réels et pour définir des règles partagées pour cadrer les activités de consommation et leur donner du sens.

Dans les rapports "défensifs », les parents prennent très au sérieux l'importance de l'argent de poche afin d'aider leurs enfants à bien consommer. Néanmoins, lorsque les parents jugent que l'enfant démontre un attachement 
exagéré et une sorte de soumission à l'univers marchand, ils considèrent que la pratique de l'argent de poche constitue une opportunité de réguler les désirs de consommation de leurs enfants. Certains parents ont trouvé, dans la pratique de l'argent de poche, une solution pour ne plus être confrontés aux demandes incessantes de l'enfant. Ils confrontent l'enfant à des arbitrages difficiles entre ses propres désirs et la réalité du montant d'argent de poche qui lui est alloué, en l'accompagnant et discutant avec lui des meilleurs choix à opérer. En revanche dans certains cas, si la médiation sémiotique relative à l'argent de poche s'avère pauvre, la pratique de celui-ci ne contribuera que très marginalement aux apprentissages de l'enfant en matière de consommation. Cette situation est plus fréquente dans le cas de rapports de type "réactifs » entre parents et enfants à propos de l'argent de poche.

D'une manière générale, nous avons constaté que les parents que nous avons interviewés considèrent que la gestion de l'argent de poche est une activité qui concerne directement et exclusivement leurs enfants. Cette rhétorique parentale mériterait certainement d'être approfondie pour révéler à quel système normatif - idéal d'un enfant mature ou de parents respectueux des choix de leurs enfants - elle fait référence. Toutefois, la déclaration «Il peut acheter ce qu'il veut avec son argent de poche » ne doit pas être comprise comme la liberté laissée à l'enfant d'utiliser son argent sans avoir à en rendre compte à ses parents. En effet, nos observations révèlent que l'utilisation de l'argent de poche par les enfants est très fortement influencée et cadrée par les conduites et les recommandations parentales. Les arguments des parents montrent qu'ils n'accordent pas à leurs enfants une liberté inconditionnelle, mais qu'ils s'assurent que le type d'achats effectués par leurs enfants est conforme aux normes sociales qui leur paraissent devoir s'imposer.

Les éléments identifiés au cours de notre enquête, montrent que la réalisation d'une grande majorité des «envies » des enfants prend place dans la perspective du projet éducatif familial. Pour participer aux activités de consommation, les enfants doivent apprendre les valeurs familiales auxquelles les différents acteurs sociaux avec lesquels ils interagissent font référence, ce qui fait que les désirs qu'ils expriment sont fortement cadrés par les valeurs et style de vie de leurs parents. Devant la pression de NR et de sa petite sœur pour recevoir des jouets de plus de 100 euros, leurs parents ont accepté de revoir le principe qu'ils avaient établi jusque-là de ne pas faire de gros cadeaux aux enfants. Toutefois, ils ont exigé que les sœurs partagent le cadeau, offert par plusieurs membres de la famille. Ainsi, le cadeau était un type de jouet qui permettait aux sœurs de jouer ensemble, dans le respect d'une de valeur 
familiale fondamentale: le partage. Après deux refus pour l'achat d'un téléphone portable, les collégiens DN et JZ (élèves de $6^{\text {ème }}$, âgés de 12 ans) l'ont remplacé par un iPod, produit qui répondait à la fois à leur quête identitaire tout en respectant les principes moraux exposés par leurs parents qui considéraient qu'ils étaient trop jeunes pour utiliser un téléphone portable.

Les interactions à propos de l'utilisation de l'argent de poche révèlent aussi que d'autres apprentissages se mettent en place au-delà de la sphère marchande. Nous avons constaté que les initiatives que prennent les enfants pour s'acheter les produits dont ils ont envie, ne sont pas uniquement liées à leur degré de développement cognitif. Comme l'a montré Lundby (2012), l'argent est souvent perçu de façon stricte par l'enfant comme un outil pour témoigner son amitié à ses pairs, à travers l'achat d'un cadeau, par exemple. De telles initiatives enfantines émergent peu à peu de l'accord ou du refus des parents de payer pour les achats de leurs enfants. En interdisant ou autorisant l'achat de certains produits, les parents mettent en valeur les signes sociaux sur lesquels les jeunes consommateurs sont en mesure de construire leurs univers de référence, correspondant à un usage socialement acceptable de l'argent de poche. Si les parents acceptent que l'enfant fasse des petits gestes pour ses amis, qu'il s'achète des livres ou offre des bonbons à ses pairs, l'impossibilité d'utiliser l'argent de poche pour acheter des produits socialement déplacés est souvent évoquée sous une forme détournée, comme une sorte de plaisanterie qui relèverait de l'absurde...

Par la mise en place d'une pratique familiale d'argent de poche, les parents soulignent que l'autonomie en matière de consommation ne consiste pas uniquement à exercer un choix parmi un ensemble de produits, mais aussi à tenir compte des valeurs familiales, des normes sociales et des limites budgétaires à imposer aux achats. Ils justifient cette pratique éducative par le fait que l'argent de poche exerce une contrainte sur les désirs de l'enfant, contribuant à l'apprentissage de la valeur de l'argent : celui-ci limite donc les demandes de consommation exprimées par l'enfant. "Ça les freine forcément, puisqu'ils sont obligés de gérer quelque chose et donc de compter, ils sont obligés de compter », confirme la mère de $\mathrm{FC}$, élève de $6^{\text {ème. }}$. Notre étude montre que l'apprentissage induit par l'utilisation de l'argent de poche, loin de favoriser l'insertion des enfants dans une culture de consommation à travers la pratique récurrente d'achats en toute indépendance, apparaît davantage comme le résultat d'une stratégie parentale visant à limiter les dépenses prescrites par les enfants. Ainsi, pour les parents, attribuer de l'argent de poche à l'enfant conduit à imposer à l'enfant la confrontation de ses envies à une limite 
financière pour aboutir in fine à une auto-régulation de ses désirs d'achat.

\section{Conclusion}

L'observation des activités relatives à l'argent de poche révèle que les conduites des jeunes consommateurs dans le monde marchand, ne résultent pas de l'indépendance des jeunes. Les enfants sont de véritables acteurs, de véritables consommateurs, mais ils reconnaissent tout à fait leur dépendance à l'égard d'autrui - et tout particulièrement de leurs parents - pour parvenir à donner du sens à leur rôle de consommateur et aux activités de consommation auxquelles ils participent.

Notre recherche confirme que la médiation parentale constitue l'élément central de la co-construction d'une autonomisation progressive de l'enfant dans l'espace marchand. Cette médiation parentale met en exergue les valeurs familiales et les normes sociales en précisant le sens de l'univers de produits accessibles aux enfants et ceux qui leur sont interdits. Notre travail souligne l'importance de la dimension intersubjective des rapports d'interaction suscités par les activités de consommation, mais également le caractère bidirectionnel du processus de développement de l'enfant qui participe activement à son propre apprentissage de consommateur.

Toutefois, les rapports établis au sein de la famille pour maîtriser les pratiques de consommation liées à l'argent de poche sont particulièrement complexes, puisque dans certaines familles, les parents établissent des rapports parfois différents avec chacun de leurs enfants en fonction de la façon dont la discussion à propos de l'argent de poche s'instaure.

Enfin, notre étude fait ressortir que la pratique de l'argent de poche n'implique pas forcément que les parents soient d'accord sur les acquisitions envisagées par leurs enfants. Véritable stratégie éducative permettant d'accompagner l'insertion de l'enfant dans une culture de consommation, l'utilisation de l'argent de poche apparaît également comme un moyen de réduire les dépenses prescrites par l'enfant.

Néanmoins, ayant progressivement constitué un échantillon de convenance pour accéder à 12 familles poitevines, la typologie que nous avons établie est par nature exploratoire et recèle d'importantes limites.

Premièrement, les résultats de notre étude dépendent des profils socioéconomiques des familles qui ont accepté d'interagir avec nous. Bien que notre typologie soit issue des rapports observés dans des catégories variées de 
famille - familles monoparentales, traditionnelles et recomposées; des familles riches, de classe moyenne et défavorisée ; des familles nombreuses, des familles avec un ou plusieurs enfants - , il n'est pas possible de généraliser nos résultats. Il semble nécessaire d'entreprendre de nouvelles recherches empiriques de façon à comprendre comment dans ces différents types de familles s'instaure le rapport entre parents et enfants à propos de l'argent de poche et comment se construisent les apprentissages liés à l'argent de poche. Ce n'est qu'ensuite qu'il pourrait s'avérer opportun de tester empiriquement sur un échantillon représentatif des familles françaises une typologie complétée et enrichie afin de construire une modélisation plus générale.

Deuxièmement, notre étude s'est limitée aux élèves de CM2 et de $6^{\text {ème }}$, ce qui souligne l'intérêt d'interagir avec des groupes d'enfants d'âges différents pour comprendre si la place dans la fratrie est susceptible de modifier l'effet de la médiation parentale. Cela permettrait également d'explorer de façon plus approfondie les ressorts que la dynamique d'autonomisation liée à la pratique de l'argent de poche instaure entre enfants et parents. Comme l'a montré de Singly (2006), les adonaissants entament un processus d'individualisation qui marque la fin de l'enfance et conduit à l'affirmation progressive de soi. L'attribution d'argent de poche couronne-t-elle des efforts d'autonomisation préalables de l'enfant dans d'autres domaines que la consommation ou bien s'accompagne-t-elle d'autres formes d'autonomisation ?

Troisièmement, des recherches comparatives internationales pourraient également être conduites (Hé, 2006) pour comprendre l'influence de facteurs culturels et institutionnels sur la signification de l'argent de poche. Il serait particulièrement intéressant de décrire et analyser les multiples distanciations qu'opère l'autonomisation des enfants dans la sphère marchande dans des pays où prédominent des logiques informelles d'éducation à la consommation par rapport à des pays où des logiques formelles d'éducation à la consommation sont portées par l'institution scolaire (Agundez Rodriguez, Samson et Jutras, 2008 ; De La Ville, 2013).

Conscients qu'il reste encore de nombreuses pistes de recherche pour comprendre le rôle que joue l'argent de poche dans le processus d'autonomisation de l'enfant dans la sphère marchande, nous formulons deux principes éducatifs susceptibles de nourrir une réflexion de la part des parents.

Pour aider leur enfant à acquérir des compétences de consommateur, les parents doivent pleinement assumer l'ambivalence qui caractérise la pratique de l'argent de poche : 
- d'une part, donner l'opportunité à leur enfant de s'initier à une pratique sociale dans une société de consommation de masse qui accorde à l'enfant le pouvoir et le droit de consommer pour trouver des ressources nécessaires à son individuation, et,

- d'autre part, manier avec subtilité l'argent de poche comme un antidote destiné à réguler les désirs enfantins de consommation en créant une situation de contrainte négociée qui conduit l'enfant à constater qu'il ne peut pas réaliser toutes ses envies, ce qui le motive à faire des arbitrages à partir de la confrontation entre ses propres désirs et la limite de son propre pouvoir d'achat.

\section{Références bibliographiques}

Agundez Rodriguez, A., Samson, G. et Jutras, F. (2008). Proposition d'un modèle d'éducation à la consommation au secondaire. VertigO - La revue électronique en sciences de l'environnement, 8 (3). doi:10.4000/vertigo.6062

Barnet-Verzat, C. et Wolff, F.-C. (2001). L'argent de poche versé aux jeunes : l'apprentissage de l'autonomie financière. Économie et statistique, 3(343), 51-72.

Baumrind, D. (1971). Current patterns of parental authority. Developmental Psychology, 4(1, Pt.2), 1-103. doi:10.1037/h0030372

Brée, J. (1993). Les enfants, la consommation et le marketing. Paris : Presses Universitaires de France.

Conseil Supérieur de l'Audiovisuel, et Crédit Agricole. (2008). Baromètre CSA/Crédit Agricole. Les parents et l'argent de poche des enfants (Sondage No. 0800828-Juillet 2008) (p. 49). Paris : Conseil Supérieur de L'audiovisuel. Récupéré le 30 octobre 2013 de http://www.csa.eu/multimedia/data/sondages/data2008/opi20080707les-parents-et-l-argent-de-poche-des-enfants.pdf

Conseil Supérieur de l'Audiovisuel et Crédit Agricole. (2009). Baromètre CSA/Crédit Agricole. Les parents et l'argent de poche des enfants (Sondage No. 0900922-V3-Juillet 2009) (p. 31). Paris : Conseil Supérieur de L'audiovisuel. Récupéré le 30 octobre 2013 de http://www.csa.eu/multimedia/data/sondages/data2009/opi20091107les-parents-et-1-argent-de-poche-des-enfants.pdf

Darling, N. et Steinberg, L. (1993). Parenting style as context : an integrative model. Psychological Bulletin, 113(3), 487-496. doi:10.1037/00332909.113.3.487 
Dias, M. (2012). L'autonomisation de l'enfant consommateur par l'activité «argent de poche»: observation participante des pratiques des écoliers de CM2 et de collégiens de 6ème (Thèse de doctorat : Sciences de gestion). Université de Poitiers.

De La Ville, V.-I. (2013). Le marketing comme «pharmakôn» : éléments pour une co-éducation à la consommation. Dans $\mathrm{P}$. Bourgne (dir.), Le marketing, poison ou remède? Les effets du marketing dans une société en crise, (p. 77-92), Colombelles : EMS.

De La Ville, V.-I. et Tartas, V. (2008). Transformer la participation de l'enfant aux activités de consommation alimentaire. Enfance, 60(3), 299-307. doi:10.3917/enf.603.0299

De La Ville, V.-I. et Tartas, V. (2011). De la socialisation du consommateur à la participation aux activités de consommation : apports de la psychologie sociohistorique et culturelle. Management \& Avenir, 42(2), 131-149. doi:10.3917/mav.042.0133

De Singly, F. (2006). Les adonaissants . Paris : Armand Colin.

Filser, M. (1994). Le comportement du consommateur. Paris : Dalloz.

Furnham, A. (1999). The saving and spending habits of young people. Journal of Economic Psychology, 20(6), 677-697. doi:10.1016/S01674870(99)00030-6

France. Ministère de la culture et de la communication. Département des études et de la prospective. Octobre, S. et Donnat, O. (2004). Les loisirs culturels des 6-14 ans. Paris : La Documentation Française.

Gentina, E. et Fosse, M.-H. (2008). L'adolescente consommatrice à la recherche de son autonomie: application au marché du maquillage. Lille, France. Récupéré le 30 octobre 2013 http://tel.archives-ouvertes.fr/tel00361520/fr/

Glaser, B. G. et Strauss, A. L. (2010). La découverte de la théorie ancrée: stratégies pour la recherche qualitative. Paris : Armand Colin (Ouvrage original publié en 1967 sous le titre The Discovery of Grounded Theory: Strategies for Qualitative Research, London, Weindenfeld and Nicolson).

Gollety, M. et Gautellier, C. (2001). Les parents, des éducateurs à la consommation, à part entière. Vers l'éducation nouvelle, (500), 20-27.

Hé, J. H. (2006). Pratiques éducatives parentales : comparaison France, Japon et Chine. La revue internationale de l'éducation familiale, 20, 9-29. doi:10.3917/rief.020.0009

Heilbrunn, B. (2004). Les pouvoirs de l'enfant consommateur. Dans F. de Singly (dir.), Enfants adultes : vers une égalité de statuts ? (p. 49-63). 
Paris : Encyclopaedia Universalis.

Honneth, A. (2008). L'autonomie décentrée. Dans M. Jouan (dir.), Psychologie morale : autonomie, responsabilité et rationalité pratique. (p. 347-363). Paris : J. Vrin.

Leontiev, A. N. (1978). Activity, consciousness, and personality. Kent : Prentice-Hall.

Lundby, E. (2012). If you have money, you can be kinder to them : possessions and economic resources in children's peer groups. Young Consumers : Insight and Ideas for Responsible Marketers, 13(2), 136-146. doi:10.1108/17473611211233477

Mackenzie, C. et Stoljar, N. (dir.) (2000). Relational autonomy, feminist perspectives on autonomy, agency, and the social self. New York, Oxford : Oxford University Press.

Mandell, N. (1988). The Least-Adult Role in Studying Children. Journal of Contemporary Ethnography, 16(4), 433-467. doi: $10.1177 / 0891241688164002$

Marshall, H. R. et Magruder, L. (1960). Relations between Parent Money Education Practices and Children's Knowledge and Use of Money. Child Development, 31(2), 253-284. doi:10.2307/1125902

Martens, L., Southerton, D. et Scott, S. (2004). Bringing children (and Parents) into the sociology of consumption towards a theoretical and empirical agenda. Journal of Consumer Culture, 4(2), 155-182. doi: $10.1177 / 1469540504043680$

McLeod, J. et O'Keefe, G. (1972). The Socialization Perspective and Communication Behavior. Dans G. F. Kline et P. J. Tichenor (dir.), Current Perspectives in Mass Communication Research (p. 121-168). Beverly Hills (Calif.) : Sage Publications.

McNeal, J. U. (1992). Kids as customers : a handbook of marketing to children. New York, N. Y : Macmillan.

McNeal, J. U. (2007). On becoming a consumer : the development of consumer behavior patterns in childhood. Amsterdam : Butterworth-Heinemann.

Montandon, C. et Sapru, S. (2002). L'étude de l'éducation dans le cadre familial et l'apport des approches interculturelles. Dans P. Dasen et C. Perregaux (dir.) Pourquoi des approches interculturelles en sciences de l'éducation? (p. 125-145). Bruxelles : De Boeck.

Moschis, G. P. (1985). The Role of Family Communication in Consumer Socialization of Children and Adolescents. Journal of Consumer Research, 11(4), 898-913.

Rogoff, B., Matusov, E. et White, C. (1996). Models of teaching and learning 
: participation in a community of learners. Dans D. R. Olson et N. Torrance (dir.) The Handbook of Education and Human Development (p. 388-414). Cambridge, (Mass.) : Blackwell Publishers.

Rogoff, B., Moore, L., Najafi, B., Dexter, A., Correa-Chávez, M. et Solis, J. (2007). Développement des répertoires culturels et participation des enfants aux pratiques quotidiennes. Dans G. Brougère et Vandenbroeck (dir.) Repenser l'éducation des jeunes enfants (p. 103-138). Bruxelles : Peter Lang.

Roland-Levy, C. (2005). L'argent de poche comme révélateur du processus de socialisation de l'enfant consommateur. Dans V.-I. De La Ville (dir.) L'enfant consommateur : variations interdisciplinaires sur l'enfant et le marché. (p. 51-71). Paris : Vuibert.

Siegel, D. L., Coffey, T. J. et Livingston, G. (2004). The great tween buying machine capturing your share of the multi-billion-dollar tween market. Chicago : Dearborn Trade Pub.

Vulbeau, A. (2007). L'argent de poche. Informations sociales, 137, 121122.

Vygotski, L. S. (1978). Mind in society : the development of higher psychological processes. Cambridge, (Mass.) : Harvard University Press.

Wertsch, J. V. (1984). The zone of proximal development : Some conceptual issues. Dans J. V Wertsch. et B. Rogoff (dir.), Children's learning in the «zone of proximal development» (p. 7-18). San Francisco : Jossey-Bass. 